\title{
ULTRAVIOLET-INDUCED AMORPHIZATION OF CUBIC ICE AND ITS IMPLICATION FOR THE EVOLUTION OF ICE GRAINS
}

\author{
A. Kouchi* and T. Kuroda \\ Institute of Low Temperature Science, Hokkaido University, \\ Sapporo 060, Japan \\ * present address \\ Laboratory Astrophysics, University of Leiden, \\ Niels Bohrweg 2, $2333 \mathrm{CA}$, Leiden, The Netherlands
}

\begin{abstract}
We found that cubic ice is transformed below $70 \mathrm{~K}$ to amorphous ice by ultraviolet irradiation, whereas no change in structure is observed at temperatures above $70 \mathrm{~K}$, regardless of the irradiation time. Experimental results can be interpreted by theoretical consideration of nucleation and growth of cubic ice in amorphous ice. We also discuss the evolution of ice grains in space on the basis of the experimental results.
\end{abstract}

\section{INTRODUCTION}

Despite current interest in effects of ultraviolet radiation and high energy particles on ice and on solid mixtures of water and other molecules, most attention has so far been paid to chemical reactions alone. On the other hand, even pure substances are affected by irradiation as was shown by Lepault et al. (1983) who found that $100 \mathrm{kV}$ electron-beam bombardment will convert ice crystals to amorphous ice below $70 \mathrm{~K}$. Accordingly, we have studied the effect of UV radiation on ice, using electron diffraction method.

Phase transformation of $\mathrm{H}_{2} \mathrm{O}$ ice at low temperature and at low pressure is especially important for understanding the evolution of ice grains in space. At temperatures lower than $140 \mathrm{~K}$, amorphous ice transforms continuously and irreversibly from one metastable state to the other with increasing temperature (Hagen et al., 1981; Kouchi, 1990). At temperatures around $140 \mathrm{~K}$, amorphous ice crystallizes to cubic ice, then cubic ice transforms to hexagonal ice by further heating. Since all these transformations are irreversible, it has so far been considered that cubic and hexagonal ice formed at high temperatures will persist, even when they have been subsequently cooled to lower temperature. However, this ignores the effect of UV radiation which is normally present in space. We found that UV irradiation transforms cubic ice into amorphous ice below $70 \mathrm{~K}$ (Kouchi and Kuroda, 1990).

\section{7}

A.C. Levasseur-Regourd and H. Hasegawa (eds.), Origin and Evolution of Interplanetary Dust, 87-90.

(C) 1991 Kluwer Academic Publishers, Printed in Japan. 


\section{EXPERIMENTAL METHOD}

A thin $\mathrm{film}$ of cubic ice was prepared at $135 \mathrm{~K}$ by vapor deposition in a vacuum chamber at a pressure of $5 \times 10^{-7} \mathrm{~Pa}$. After deposition, the cubic ice was cooled to the desired temperature and was irradiated with UV radiation produced by a $D_{2}$ lamp $\left(110<\lambda<400 \mathrm{~nm} ; \Phi \mathrm{uv} \sim 10^{12}\right.$ photon $\left.\mathrm{cm}^{-2} \mathrm{~s}^{-1}\right)$. Structural changes in the ice were examined in-situ by reflection electron diffraction $(20 \mathrm{kV})$.

\section{RESULTS}

It is found that cubic ice at temeratures lower than $70 \mathrm{~K}$ transforms to amorphous ice by UV irradiation, whereas no change in structure occurs at temperatures above $70 \mathrm{~K}$ regardless of the irradiation time. At temperatures between 50 and $70 \mathrm{~K}$, irradiation for 30 to $60 \mathrm{~min}$ was required for amorphization, whereas only several tens of seconds were required at $10 \mathrm{~K}$. Figure 1 shows reflection electron diffraction patterns of cubic ice and UV-induced amorphous ice. The halo diffraction pattern in Fig. Ib indicates that the irradiated ice is amorphous.

Lepault et al. (1983) found that crystalline ice was transformed below $70 \mathrm{~K}$ into amorphous ice after irradiation with $100 \mathrm{kV}$ electrons. However, we did not observe structural change after irradiation with 30 $\mathrm{kV}$ electrons (maximum energy of our electron gun) even at $10 \mathrm{~K}$. We were not able to exclude amorphization with $30 \mathrm{kV}$ electrons even at 10 $k$. The only explanation for this was that the electron flux was too low, but the need for further investigation is indicated.

Our finding of amorphization by UV-irradiation is supported by the latest measurements of infrared absorbance spectra (Kouchi and Greenberg, in prep.). Spectral features and the peak frequency of the $0 \mathrm{H}-\mathrm{stretching}$ mode of cubic ice around $3 \mu \mathrm{m}$ are substantially changed by the UV irradiation. The structure of the UV-induced amorphous ice absorption is similar to that of the amorphous ice which was directly obtained by vapor deposition at $10 \mathrm{~K}$.
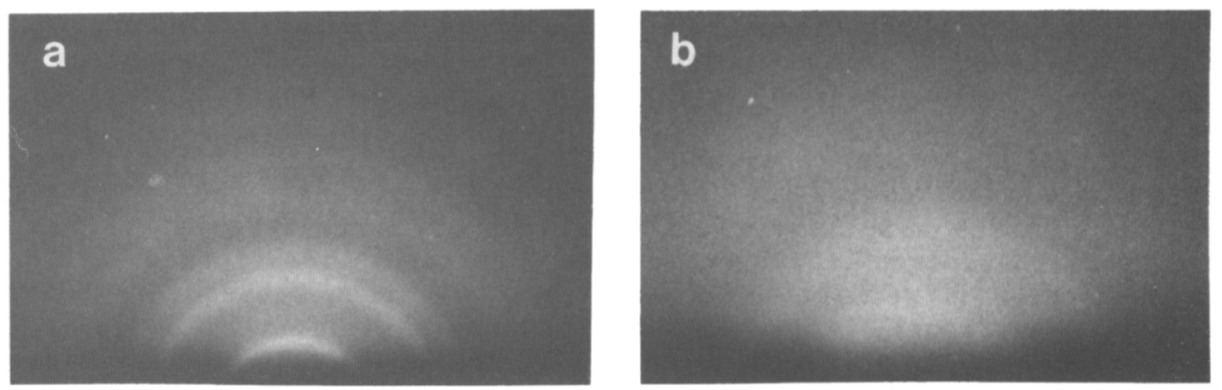

Figure 1. Reflection electron diffraction patterns of a) cubic ice produced at $135 \mathrm{~K}$, and b) UV-induced amorphous ice after $10 \mathrm{~min}$ of irradiation of cubic ice at $10 \mathrm{~K}$. 


\section{THEORETICAL CONSIDERATION}

Here we briefly mention some theoretical aspects of amorphization. There are two competitive processes relevant to amorphization by irradiation. The first one is the process of destroying the cubic lattice caused by displacement or dissociation of molecules by irradiation, and the second one is the restoration process from unstable amorphous structure to stable crystal structure leading to lowering of the free energy of the system. The latter process is controlled by the rate $J$ of nucleation of stable crystalline clusters and their growth rate $v$. The self-diffusion coefficient $D a$ of amorphous ice is involved with $J$ and $v$ through the rearrangement of molecules from amorphous to crystalline structure. Accordingly, the restoration process is exponentially activated with increasing temperature, so that the resistance of ice to amorphization by irradiation is lost at a critical temperature $\mathrm{TC}$. In order to quantitatively understand $\mathrm{Tc}$, measurement of $\mathrm{Da}$ and the interfacial free energy $\gamma$ between amorphous and cubic ice are required. It is to be noted that there are several forms of metastable amorphous structures (Kouchi, 1990), and the values of Da and $r$ may depend on the structure. Thus, strictly speaking, Tc may depend on experimental conditions.

\section{ASTROPHYSICAL IMPLICATION}

Our results have several implications for ice grains in space. The interstellar UV radiation fluxes in diffuse and dense molecular clouds are $10^{8}$ photon $\mathrm{cm}^{-2} \mathrm{~s}^{-1}$ (Hagen et al., 1979) and 103-4 (Prasad and Tarafdar, 1983), respectively. A UV flux of $10^{4}$ photon $\mathrm{cm}^{-2} \mathrm{~s}^{-1}$ in dense clouds is probably more relevant when including cosmic ray, solar wind and penetrating sources together. The time scale equivalent to one hour of irradiation in the laboratory is about 1 year in the diffuse medium and $10^{4}$ years in a dense cloud. We may conclude that if grains of cubic ice or of amorphous ice are formed at around $100 \mathrm{~K}$ in a stellar atmosphere (Seki and Hasegawa, 1983), they will be transformed by UV irradiation to amorphous ice of low temperature form (Kouchi and Greenberg, in prep.) in an astronomically short time after cooling to 10 $K$ after injection into space.

\section{ACKNOWLEDGMENTS}

We thank Professors H. Hasegawa for discussions and J.M. Greenberg for discussions and critical reading of the manuscript. This work was partly supported by a Grant-in-Aid for scientific research on Priority Area (Origin of the Solar System) from the Japanese Ministry of Education, Science and Culture, and by the Yamada Science Foundation, Japan .

\section{REFERENCES}

Hagen, W., Allamandola, L.J. and Greenberg, J.M. (1979) 'Interstellar molecule formation in grain mantles: the laboratory analog 
experiments, results and implications', Astrophys. Space Sci.,65, 215240 .

Hagen, W., Tielens, A.G.G.M. and Greenberg, J.M. (1981) 'The infrared spectra of amorphous solid water and Ic between 10 and $140 \mathrm{~K}^{\prime}$, Chem. Phys., 56, 367-379.

Kouchi, A. (1990) 'Evaporation of $\mathrm{H}_{2} \mathrm{O}-\mathrm{CO}$ ice and its astrophysical implications', J. Cryst. Growth, 99, 1220-1226.

Kouchi, A. and Kuroda, T. (1990) 'Amorphization of cubic ice by ultraviolet irradiation', Nature, 344, 134-135.

Lepault, J., Freeman, R. and Dubochet, J. (1983) 'Electronbeam induced vitrified ice', J. Microsc., 132, RP3-4.

Prasad, S.S. and Tarafdar, S.P. (1983) 'UV radiation field inside dense clouds: its possible existence and chemical implications', Astrophys. J., 267, 603-609.

Seki, J. and Hasegawa, H. (1983) The heterogeneous condensation of interstellar ice grains', Astrophys. Space Sci., 94, 177-189. 